\title{
Überzeugtes JA der Ärzteschaft zur Tarifrevision!
}

\section{Urs Stoffel}

Dr. med., Mitglied des Zentralvorstandes, Departementsverantwortlicher Ambulante Versorgung und Tarife

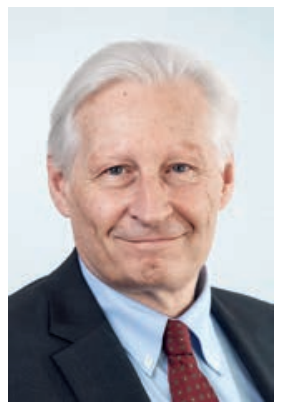

Bereits im Mai 2018 hat die Ärztekammer mit TARCO die FMH-intern erarbeitete revidierte Leistungsstruktur einstimmig verabschiedet. Das interne Projekt TARCO wurde damit abgeschlossen.

Inzwischen hat die FMH mit den Tarifpartnern die gemeinsame Revision der Leistungsstruktur verhandelt. Die Ergebnisse des Projekts TARCO bildeten dabei die Vorgaben für die Verhandlungen mit den Tarifpartnern. Nach anspruchsvollen und intensiven Verhandlungen gelang es uns, den überwiegenden Teil der TARCO-Resultate in die neue gemeinsame Leistungsstruktur zu integrieren. Gestern haben alle Tarifpartner der ats-tms in einem historischen Akt zusammen

\section{Es gibt noch viel zu tun - packen wir es gemeinsam an!}

über die jetzt vorliegende, gemeinsam revidierte Leistungsstruktur kommuniziert. Nach jahrelanger Blockade ist es dank der Bereitschaft aller beteiligten Tarifpartner damit gelungen, den Durchbruch für eine gemeinsame Lösung zu schaffen.

Diese sachgerechte und betriebswirtschaftlich bemessene Leistungsstruktur (gemäss Art. 43, Abs. 4 KVG) ist das unverzichtbare Herzstück eines Einzelleistungstarifs.

Bereits am 25. Oktober 2018 hat die Ärztekammer auf Empfehlung der Delegiertenversammlung der FMH mit überwältigender Mehrheit dieser verhandelten Leistungsstruktur zugestimmt und damit klar Verantwortung übernommen.

Selbstredend ist klar, dass in einem Verhandlungstarif nicht alle Vorgaben und Ziele der einzelnen Tarifpartner erfüllt werden können. Es braucht Konsensund Kompromissbereitschaft, um das gemeinsame Ziel einer Tarifpartnerschaft nicht zu gefährden. Gelingt dies nicht, so ist die unvermeidliche Alternative eine Fortsetzung des Amtstarifs.

Trotzdem ist uns ebenso bewusst, dass die unvermeidbaren Kompromisse, welche bei einzelnen Tarifkapiteln eingegangen werden mussten, für manche Fachgesell- schaften mitunter schmerzliche und einschneidende Einbussen bedeuten können. Ebenso ist klar, dass sich bei der vorliegenden Version der Leistungsstruktur noch offensichtliche Fehler und Falschinterpretationen eingeschlichen haben, welche in der Etappe zwei des Projekts gemeinsam mit den Tarifpartnern noch korrigiert werden müssen.

\section{Wie geht es nun weiter?}

Nachdem nun alle Tarifpartner der ats-tms (Curafutura, MTK und FMH) der vorliegenden Leistungsstruktur zugestimmt haben, kann auf Ende Jahr dem Bundesrat eine tarifpartnerschaftliche, revidierte Leistungsstruktur (noch ohne Verträge und Regelwerk) zur Kenntnisnahme eingereicht werden.

In der bereits begonnenen Etappe zwei sollen nun das zur Leistungsstruktur gehörende Regelwerk mit Limitationen, Kumulationen etc. sowie auch die Verträge mit Anhängen fertig verhandelt werden. Zur Festlegung der Abrechnungsregeln (Limitationen, Kumulationen etc.) werden nochmals alle Fachgesellschaftsvertreter zusammen mit den FMH-Experten und den Tarifpartnern am Verhandlungstisch sitzen. Dieser enorm zeitaufwendige Prozess mit über 30 geplanten Workshops ist wichtig und notwendig, um bei den

\section{In einem historischen Akt haben gestern} alle ats-tms-Tarifpartner der vorliegenden gemeinsam revidierten Leistungsstruktur zugestimmt.

Fachgesellschaften die nötige Akzeptanz und Transparenz für die Tarifrevision zu schaffen. Ohne das ärztliche Know-how in diesem Bereich kann keine sachgerechte Tarifstruktur geschaffen werden.

Ziel ist und bleibt es, im Frühsommer 2019 dem Bundesrat ein gemeinsames und tarifpartnerschaftlich verhandeltes Gesamtpaket einer einheitlichen Einzelleistungstarifstruktur zur Prüfung und Festsetzung einzureichen. 